\title{
Prevention of Alzheimer's disease: Omega-3 fatty acid and phenolic anti-oxidant interventions
}

\author{
Greg M. Cole ${ }^{a, b, c, *}$, Giselle P. Lim ${ }^{a, b}$, Fusheng Yang ${ }^{a, b}$, Bruce Teter ${ }^{a, b}$, Aynun Begum ${ }^{a, b}$, \\ Qiulan $\mathrm{Ma}^{\mathrm{a}}{ }^{\mathrm{a} b}$, Marni E. Harris-White ${ }^{\mathrm{a}, \mathrm{b}}$, Sally A. Frautschy ${ }^{\mathrm{a}, \mathrm{b}, \mathrm{c}}$ \\ ${ }^{a}$ Greater Los Angeles Veterans Affairs Healthcare System, Geriatric Research, Education and Clinical Center, Sepulveda, CA 91343, USA \\ ${ }^{\mathrm{b}}$ Department of Medicine, University of California, Los Angeles, CA 90095, USA \\ ${ }^{c}$ Department of Neurology, University of California, Los Angeles, CA 90095, USA
}

Received 18 August 2005; accepted 5 September 2005

\begin{abstract}
Alzheimer's disease (AD) and cardiovascular disease (CVD) are syndromes of aging that share analogous lesions and risk factors, involving lipoproteins, oxidative damage and inflammation. Unlike in CVD, in $\mathrm{AD}$, sensitive biomarkers are unknown, and high-risk groups are understudied. To identify potential prevention strategies in $\mathrm{AD}$, we have focused on pre-clinical models (transgenic and amyloid infusion models), testing dietary/lifestyle factors strongly implicated in reducing risk in epidemiological studies. Initially, we reported the impact of non-steroidal anti-inflammatory drugs (NSAIDs), notably ibuprofen, which reduced amyloid accumulation, but suppressed few inflammatory markers and without reducing oxidative damage. Safety concerns with chronic NSAIDs led to a screen of alternative NSAIDs and identification of the phenolic anti-inflammatory/anti-oxidant compound curcumin, the yellow pigment in turmeric that we found targeted multiple AD pathogenic cascades. The dietary omega-3 fatty acid, docosahexaenoic acid (DHA), also limited amyloid, oxidative damage and synaptic and cognitive deficits in a transgenic mouse model. Both DHA and curcumin have favorable safety profiles, epidemiology and efficacy, and may exert general anti-aging benefits (anti-cancer and cardioprotective.)
\end{abstract}

(C) 2005 Elsevier Inc. All rights reserved.

Keywords: Docosahexaenoic acid; Curcumin; Alzheimer's; Prevention; Anti-oxidant; Anti-inflammatory

\section{Introduction}

Alzheimer's disease (AD) resembles cardiovascular disease (CVD) since both show age-dependent accumulation of lipophilic material in multicellular plaque lesions, which involves a macrophage lineage (microglial) inflammatory response, oxidative damage and injury to surrounding cells. The genetic (ApoE alleles) and environmental (high homocysteine, statin use/cholesterol/inflammatory markers/dietary fats/fish/wine/type II diabetes, low exercise, etc.) risk factors for $\mathrm{AD}$ and CVD show strong overlap, suggesting that prevention approaches for reducing CVD may be relevant

\footnotetext{
* Corresponding author. Present address: Greater Los Angeles Healthcare System, Veterans Administration Medical Center, 16111 Plummer St., Building 7, Room A102, North Hills, CA 91343, USA.

Tel.: +1 818891 7711x9949; fax: +1 8188955835 .

E-mail address: gmcole@ucla.edu (G.M. Cole).
}

to AD. Studies with CVD in both animal models and the clinic have identified relevant epidemiological risk factors that can be applied to development of successful prevention of CVD. Two major advantages for CVD prevention have been adequate biomarkers (blood lipid profiles) and the opportunity to conduct prevention trials in at-risk patients with secondary cardiovascular "events" as endpoints. Unfortunately, the lack of suitable biomarkers, "second events" and the cost and time involved in conducting prevention trials for AD have prohibited testing prevention in clinical trials; most testing has been in $\mathrm{AD}$ patients. Clinical trials in $\mathrm{AD}$ patients with vitamin $\mathrm{E}$, and several NSAIDs (naproxen, cyclo-oxygenase-2 (COX-2) inhibitors) have shown mostly minimal or no effects on cognitive decline and $\mathrm{AD}$ progression. These failures emphasized the need for better screening in preclinical animal models (expressing familial AD genes or after amyloid peptide infusion into the central nervous system, CNS) using drugs with 
good epidemiology and proven safety. Amyloid models have permitted testing interventions limiting $\beta$-amyloid and associated oxidative damage and inflammation. Similar studies in tau transgenics to limit tau/tangle pathology are underway.

CNS inflammation in $\mathrm{AD}$ is characterized by reactive microglia and elevated IL- 1 and complement factors [3]. A $\beta$ aggregates can stimulate oxidation in multiple ways: including neuronal $\mathrm{H}_{2} \mathrm{O}_{2}$ production [4], radical production via iron or copper binding [22] and pro-inflammatory stimulation of peroxynitrite or superoxide. Both oxidative damage $[4,24,28]$ and inflammation [3] begin early in $\mathrm{AD}$ accompanying amyloid accumulation and neurodegeneration. AD risk is reduced by anti-oxidant $[20,34]$ and non-steroidal anti-inflammatory drug (NSAID) [11] intake suggesting these might be useful prevention methods.

We initially tested the NSAID (ibuprofen) that had the strongest epidemiological rationale. With low dosing designed to model apparently protective chronic NSAID consumption in populations with reduced AD risk, ibuprofen suppressed amyloid accumulation in APPsw transgenic mice [16] but reduced a surprisingly limited subset of inflammatory markers, notably IL-1 $\beta$ and downstream murine ACT mRNA, but not iNOS, macrosialin or CD11c mRNA [19]. Further, some NSAIDs including ibuprofen (but not naproxen and $\mathrm{COX}-2$ inhibitors) selectively lowered $\mathrm{A} \beta 1-42$ production without inhibiting $A \beta 1-40$ or NOTCH in vitro or in vivo [8,32]. Although ibuprofen has the strongest rationale for use in $\mathrm{AD}$ prevention, it has not been tested (side-effect concerns halted COX-2 inhibitor and naproxen prevention trials in 2004). Efficacy (and possibly even safety) issues with Vitamin E leaves us with few viable approaches to chronic control of oxidative damage and inflammation in $\mathrm{AD}$ and other diseases of aging.

\section{Phenolic anti-oxidants}

Many potent dietary anti-oxidants occur in plant fruiting bodies, seeds or roots and contain one or more phenol groups that contribute to potent anti-oxidant activities presumably selected to protect the plant germ line. Grapes, apples, many types of berries, pomegranates, green tea and many other plant sources are rich sources of these anti-oxidant compounds that often have potent anti-oxidant and antiinflammatory properties and related health benefits. Notable among these are resveratrol from red wine, the green tea catechins and the turmeric extract curcumin which have received intensive study for potential disease prevention or treatment and are worthy of consideration for AD.

\section{Curcumin is a combined polyphenolic anti-oxidant/NSAID that targets AD pathogenesis at multiple sites}

Chronic oxidative damage and inflammation occur in $\mathrm{AD}$ and likely contribute to neurodegeneration. Although other groups have some evidence for protection, in our hands, neither ibuprofen nor Vitamin E supplements reduced protein carbonyls, one marker for oxidative damage. In contrast, a polyphenolic tumeric component, diferulomethane (curcumin) lowered F2 isoprostanes and carbonyls as measures of oxidative damage, reduced IL- $1 \beta$, iNOS and CD11b as markers of activated microglia and limited cognitive deficits, postsynaptic marker loss and amyloid accumulation $[9,15]$.

Curcumin has the potential to suppress the AD pathogenic cascade at multiple sites. Curcumin's structure is two methoxyphenol groups separated by a $\beta$-diketone bridge that confers iron and other metal chelating activity. Metals have been implicated in $\mathrm{A} \beta$ aggregation and toxicity. Thus, while curcumin is a direct radical scavenger, it is a more potent anti-oxidant and inhibitor of lipid peroxidation, particularly metal catalyzed peroxidation [29]. Curcumin is also a good inhibitor of expression of inflammatory cytokines, COX-2, and iNOS by virtue of inhibition of JNK/AP-1 and NFK-Bmediated gene transcription [1]. All of these factors (IL-1, TNF $\alpha$, COX-2, iNOS, JNK, NFK-B) have been implicated in $A \beta$ toxicity or $A \beta$-induced $A D$ pathogenesis in different $\mathrm{AD}$ models, indicating potential as a multi-target intervention. Beyond this, curcumin has other anti-amyloid activities. Because curcumin is a good inhibitor of cytokines, it may also limit inflammatory and oxidative damage induction of BACE1, the $\beta$-secretase enzyme that makes the initial step in amyloid production [12]. Our data suggest that, under conditions of elevated oxidative damage and inflammation, curcumin can reduce BACE1 expression in vivo. Curcumin is not simply immunosuppressive, but immunomodulatory, simultaneously inhibiting cytokine and microglial activation indices related to neurotoxicity, but increasing mRNA and immunostaining for both $\mathrm{CD} 11 \mathrm{c}$ and macrosialin, markers for microglial phagocytosis that may clear amyloid [Cole and Morihara, unpublished data]. As previously, reviewed, curcumin's hydroxyls provide polar groups $19 \AA$ apart, resembling chrysamine $\mathrm{G}$ and the amyloid binding dye Congo red. Thus, curcumin binds and labels plaques in vitro and in vivo and inhibits amyloid aggregation in vitro and amyloid accumulation in vivo [33]. Curcumin also inhibits neurotoxic $A \beta$ oligomer formation and oligomer-dependent $A \beta$ toxicity in vitro [33]. Curcumin's multiple anti-amyloid activities may contribute to the suppression of amyloid in vivo and it's antiamyloid activity remains effective in aged mice, even after amyloid deposits are well established. Since this compound has multiple direct and indirect anti-amyloid actions including metal chelating, phagocytosis enhancing, anti-oxidant and cholesterol-lowering activity (reviewed in refs. [15,33] and possible suppression of BACE1 induction, it is likely that no one mechanism is involved. Curcumin also inhibits known amyloid response pathways including JNK kinase and iNOS expression in vitro $[5,12,23]$ and in vivo $[10,13]$ and in CNS (our data). Thus, curcumin should be able to suppress not only $A \beta$ but key aspects of the response to $A \beta$ that are JNK- and iNOS-dependent including LTP inhibition [30], reported to depend on JNK, iNOS, and microglial radicals 
[31]. With safety and so many potentially relevant therapeutic activities, curcumin has to be a strong candidate for AD prevention or treatment. While we initially screened many anti-oxidants, including several polyphenolics in an $\mathrm{A} \beta-$ infusion model and selected curcumin as the most promising candidate, one might expect utility from other dietary polyphenolics.

\section{Omega-3 fatty acids}

Neurons and synapses are highly enriched in long chain unsaturated fatty acids, the most vulnerable of these being the omega-3 fatty acid, docosahexaenoic acid (DHA, C22:6(n-3)) with six double bonds. Synapse loss is a close correlate of cognitive decline in $\mathrm{AD}$, and synapses are sites of $\mathrm{A} \beta$ generation and accumulation. DHA is oxidized in $\mathrm{AD}$ to produce elevated $\mathrm{F} 4$-isoprostanes or neuroprostanes [18]. Because DHA is heavily concentrated in neurons, its oxidation products are a good index of neuronal oxidative damage.

Fatty fish are the major dietary source of DHA, and reduced fish or DHA intake increases risk for AD [17]. Aged Tg2576 APPsw transgene-positive mice placed from 17 to 22 months on a safflower oil rich, DHA-depleting diet exhibited increased oxidative damage and transgenedependent loss of CNS DHA and massive (70-95\%) loss of postsynaptic proteins like the actin-regulatory drebrin, a dendritic spine protein known to be 70-90\% lost in AD [7,27] and NR2b [6]. Greater depletion of DHA in Tg2576 may be driven by $A \beta$ aggregates causing DHA oxidation. DHA depletion also caused transgene-dependent caspase activation and deficits in the neuroprotective "insulin signaling pathway" (PI3-kinase > Akt) similar to AD. Large deficits observed in the PI3-Kp85 $\alpha$ subunit observed with high safflower oil were only partially explained by mRNA loss and may be secondary to caspase activation. While DHA only partially suppressed $\mathrm{p} 85 \alpha$ subunit loss, it still improved neuroprotective AKT, BAD and GSK3 $\beta$ phosphorylation (unpublished results), likely through facilitation of AKT translocation and activation [2]. Although we did not detect reduced inflammatory cytokines in DHA fed AD model mice, the arachidonic acid levels were significantly reduced, suggesting potential NSAID-like activity of dietary omega-3 to reduce pro-inflammatory prostaglandin intermediates. Other AD-relevant DHA actions may include effects on metabolism to form neuroprotectin D1, an anti-apoptotic DHA metabolite that is dramatically reduced in AD [21].

DHA depletion aggravated cognitive deficits in the Morris Water Maze in the Tg2576 mouse, while DHA supplementation was protective [7] and reduced amyloid, even late in life [14]. Anti-amyloid activities include modulation of secretase activities via membrane "fluidity"/protein mobility changes or Akt $>$ GSK regulation of $\gamma$-secretase [25] and induction of anti-amyloidogenic transthyretin [26]. Like fish oil, DHA from algal sources can be safely taken as a supplement at high doses. All of DHA's neuroprotective effects may also be relevant to cognitive decline in normal aging. Both DHA and EPA are also likely protective against vascular disease, one of the other major causes of age-related dementia. Since both the cost and toxicity issues are minimal, marine omega-3 lipids are suitable for primary prevention.

\section{Conclusions}

Age-related diseases frequently cause oxidative damage and inflammation that should be preventable, but plausible anti-oxidants and anti-inflammatory approaches have not proven useful. In particular, recent data with anti-oxidants like Vitamin E and NSAIDs, including the COX-2 inhibitors and naproxen, have raised concerns about both their chronic safety and efficacy. Because of its potent anti-carcinogenic activity, curcumin has already been through extensive preclinical toxicology and clinical testing and has a very favorable safety profile $[1,13]$. Because of its availability and low cost, coupled with preclinical data showing its potential for intervention at multiple sites in AD pathogenesis, curcumin is now in clinical trials in mild to moderate $\mathrm{AD}$ patients under an FDA approved IND by the UCLA Alzheimer Center. Omega3 fatty acids have an even stronger safety profile, a long history of use and proven health benefits for CVD, yet typical Western diets have $<30 \%$ of the roughly $200-300 \mathrm{mg}$ of DHA recommended by expert panels. Epidemiology shows risk reduction of $60 \%$ associated with modest increases in DHA intake or plasma levels. DHA works well in slowing $\mathrm{AD}$ pathogenesis in mice with a human $\mathrm{AD}$ gene and is safe enough to include in infant formula. It should be a strong candidate for use in primary prevention.

\section{Acknowledgments}

This study was supported by AG13471 (GMC), NS43946 (GMC), VA Merit and Alzheimer Association (GMC), AG10685 (SAF), AG021975 (SAF).

\section{References}

[1] Aggarwal BB, Kumar A, Bharti AC. Anticancer potential of curcumin: preclinical and clinical studies. Anticancer Res 2003;23:363-98.

[2] Akbar M, Kim HY. Protective effects of docosahexaenoic acid in staurosporine-induced apoptosis: involvement of phosphatidylinositol-3 kinase pathway. J Neurochem 2002;82:655-65.

[3] Akiyama H, Barger S, Barnum S, Bradt B, Bauer J, Cole GM, et al. Inflammation and Alzheimer's disease. Neurobiol Aging 2000;21:383-421.

[4] Behl C, Davis JB, Lesley R, Schubert D. Hydrogen peroxide mediates amyloid $\beta$-protein toxicity. Cell 1994;77:817-27.

[5] Brouet I, Ohshima H. Curcumin, an anti-tumour promoter and anti-inflammatory agent, inhibits induction of nitric oxide synthase in activated macrophages. Biochem Biophys Res Commun 1995;206:533-40. 
[6] Calon F, Lim GP, Morihara T, Yang F, Ubeda O, Salem NJ, et al. Dietary $n-3$ polyunsaturated fatty acid depletion activates caspases and decreases NMDA receptors in the brain of a transgenic mouse model of Alzheimer's disease. Eur J Neurosci 2005;22:617-26.

[7] Calon F, Lim GP, Yang F, Morihara T, Teter B, Ubeda O, et al. Docosahexaenoic acid protects from dendritic pathology in an Alzheimer's disease mouse model. Neuron 2004:43.

[8] Eriksen JL, Sagi SA, Smith TE, Weggen S, Das P, McLendon DC, et al. NSAIDs and enantiomers of flurbiprofen target gamma-secretase and lower Abeta 42 in vivo. J Clin Invest 2003;112:440-9.

[9] Frautschy SA, Hu W, Miller SA, Kim P, Harris-White ME, Cole GM. Phenolic anti-inflammatory antioxidant reversal of A $\beta$ induced cognitive deficits and neuropathology. Neurobiol Aging 2001;22:991-1003.

[10] Hofman MA, Swaab DF. Alterations in circadian rhythmicity of the vasopressin-expressing neurons in the human suprachiasmatic nucleus with aging. Brain Res 1994;651:134-42.

[11] in t' Veld BA, Ruitenberg A, Hofman A, Launer LJ, van Duijn CM, Stijnen T, et al. Nonsteroidal antiinflammatory drugs and the risk of Alzheimer's disease. N Engl J Med 2001;345(21):1515-21.

[12] Kakar SS, Roy D. Curcumin inhibits TPA induced expression of c-fos, c-jun and c-myc proto-oncogenes messenger RNAs in mouse skin. Cancer Lett 1994;87:85-9.

[13] Kelloff GJ, Crowell JA, Hawk ET, Steele VE, Lubet RA, Boone $\mathrm{CW}$, et al. Strategy and planning for chemopreventive drug development: clinical development plan: curcumin. J Cell Biochem Suppl 1996;26:72-85.

[14] Lim GP, Calon F, Morihara T, Yang F, Teter B, Ubeda O, et al. A diet enriched with the omega-3 fatty acid docosahexaenoic acid reduces amyloid burden in an aged Alzheimer mouse model. J Neurosci 2005;25:3032-40.

[15] Lim GP, Chu T, Yang F, Beech W, Frautschy SA, Cole GM. The curry spice curcumin reduces oxidative damage and amyloid pathology in an Alzheimer transgenic mouse. J Neurosci 2001;21:8370-7.

[16] Lim GP, Yang F, Chu T, Chen P, Beech W, Teter B, et al. Ibuprofen suppresses plaque pathology and inflammation in a mouse model for Alzheimer's disease. J Neurosci 2000;20(15):5709-14.

[17] Maclean CH, Issa AM, Newberry SJ, Mojica WA, Morton SC, Garland $\mathrm{RH}$, et al. Effects of omega-3 fatty acids on cognitive function with aging, dementia, and neurological diseases. Evid Rep Technol Assess (Summ) 2005:1-3.

[18] Montine TJ, Quinn JF, Milatovic D, Silbert LC, Dang T, Sanchez $\mathrm{S}$, et al. Peripheral F2-isoprostanes and F4-neuroprostanes are not increased in Alzheimer's disease. Ann Neurol 2002;52:175-9.

[19] Morihara T, Teter B, Yang F, Lim GP, Boudinot S, Boudinot FD, et al. Ibuprofen suppresses interleukin-1beta induction of proamyloidogenic alpha1-antichymotrypsin to ameliorate beta-amyloid (Abeta) pathology in Alzheimer's models. Neuropsychopharmacology 2005;30:1111-20.

[20] Morris MC, Evans DA, Bienias JL, Tangney CC, Bennett DA, Aggarwal N, et al. Dietary intake of antioxidant nutrients and the risk of incident Alzheimer disease in a biracial community study. JAMA 2002;287:33230-7.
[21] Lukiw WJ, Cui JG, Marcheselli VL, Bodker M, Botkjaer A, Gotlinger $\mathrm{K}$, et al. A role for docosahexaenoic acid-derived neuroprotectin D1 in neural cell survival and Alzheimer disease. J Clin Invest 2005;115(10):2774-83.

[22] Opazo C, Huang X, Cherny RA, Moir RD, Roher AE, White AR, et al. Metalloenzyme-like activity of Alzheimer's disease beta-amyloid. $\mathrm{Cu}$-dependent catalytic conversion of dopamine, cholesterol, and biological reducing agents to neurotoxic $\mathrm{H}(2) \mathrm{O}(2)$. J Biol Chem 2002;277:40302-8.

[23] Pan MH, Lin-Shiau SY, Lin JK. Comparative studies on the suppression of nitric oxide synthase by curcumin and its hydrogenated metabolites through down-regulation of IkappaB kinase and NFkappa B in macrophages. Biochem Pharmacol 2000;60:1665-76.

[24] Pappolla MA, Smith MA, Bryant-Thomas T, Bazan N, Petanceska S, Perry G, et al. Cholesterol, oxidative stress, and Alzheimer's disease: expanding the horizons of pathogenesis. Free Radical Biol Med 2002;33:173-81

[25] Phiel CJ, Wilson CA, Lee VM, Klein PS. GSK-3alpha regulates production of Alzheimer's disease amyloid-beta peptides. Nature 2003;423:435-9.

[26] Puskas LG, Kitajka K, Nyakas C, Barcelo-Coblijn G, Farkas T. Short-term administration of omega 3 fatty acids from fish oil results in increased transthyretin transcription in old rat hippocampus. Proc Natl Acad Sci USA 2003.

[27] Shim KS, Drebrin LG. A dendritic spine protein, is manifold decreased in brains of patients with Alzheimer's disease and Down syndrome. Neurosci Lett 2002;324:209-12.

[28] Smith MA, Richey Harris PL, Sayre LM, Beckman JS, Perry G. Widespread peroxynitrite-mediated damage in Alzheimer's disease. J Neurosci 1997;17:2653-7.

[29] Venkatesan P, Rao MNA. Structure-activity relationships for the inhibition of lipid peroxidation and the scavenging of free radicals by synthetic symmetrical curcumin analogues. J Pharm Pharmacol 2000;52:1123-8.

[30] Walsh DM, Klyubin I, Fadeeva JV, Cullen WK, Anwyl R, Wolfe MS, et al. Naturally secreted oligomers of amyloid beta protein potently inhibit hippocampal long-term potentiation in vivo. Nature 2002;416:535-9.

[31] Wang Q, Rowan MJ, Anwyl R. Beta-amyloid-mediated inhibition of NMDA receptor-dependent long-term potentiation induction involves activation of microglia and stimulation of inducible nitric oxide synthase and superoxide. J Neurosci 2004;24:6049-56.

[32] Weggen S, Eriksen JL, Das P, Sagi SA, Wang R, Pietrzik CU, et al. A subset of NSAIDs lower amyloidogenic Abeta42 independently of cyclooxygenase activity. Nature 2001;414:212-6.

[33] Yang F, Lim GP, Begum AN, Ubeda OJ, Simmons MR, Ambegaokar SS, et al. Curcumin inhibits formation of amyloid beta oligomers and fibrils, binds plaques, and reduces amyloid in vivo. J Biol Chem 2005;280:5892-901.

[34] Zandi PP, Anthony JC, Khachaturian AS, Stone SV, Gustafson D, Tschanz JT, et al. Reduced risk of Alzheimer disease in users of antioxidant vitamin supplements: the Cache County Study. Arch Neurol 2004;61:82-8. 